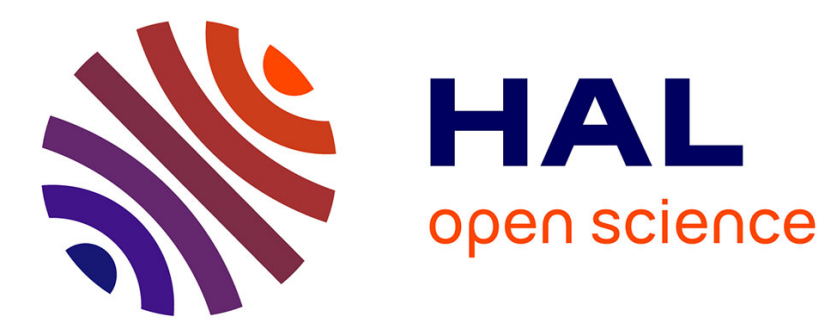

\title{
Micromirrors with controlled amplitude and phase
}

Julien Lumeau, Cihan Koc, Thomas Begou

\section{To cite this version:}

Julien Lumeau, Cihan Koc, Thomas Begou. Micromirrors with controlled amplitude and phase. Applied optics, 2017, 56 (20), pp.5655-5660. 10.1364/AO.56.005655 . hal-01558472

\section{HAL Id: hal-01558472 \\ https://hal.science/hal-01558472}

Submitted on 13 Mar 2019

HAL is a multi-disciplinary open access archive for the deposit and dissemination of scientific research documents, whether they are published or not. The documents may come from teaching and research institutions in France or abroad, or from public or private research centers.
L'archive ouverte pluridisciplinaire HAL, est destinée au dépôt et à la diffusion de documents scientifiques de niveau recherche, publiés ou non, émanant des établissements d'enseignement et de recherche français ou étrangers, des laboratoires publics ou privés. 


\title{
Micro-mirrors with controlled amplitude and phase
}

\author{
Julien lumeau ${ }^{1, *}$, Cihan Koc ${ }^{1}$ and Thomas Begou ${ }^{1}$ \\ ${ }^{1}$ Aix Marseille Univ, CNRS, Centrale Marseille, Institut Fresnel, F-13013 Marseille, France \\ *Corresponding author: julien.lumeau@fresnel.fr
}

Received XX Month XXXX; revised XX Month, XXXX; accepted XX Month XXXX; posted XX Month XXXX (Doc. ID XXXXX); published XX Month XXXX

This paper describes the fabrication of circular micro-mirrors with diameters between 50 and $150 \mu \mathrm{m}$ with controlled amplitude and phase. Design, manufacturing and characterization method are presented.

OCIS codes: (310.0310) Thin films; (350.5030) Phase; (310.1620) Interference coatings.

http://dx.doi.org/10.1364/AO.99.099999

\section{Introduction}

Optical coatings play a key role in most of the optical systems as they offer an efficient and versatile way to control the spectral properties of an incoming radiation. With the recent improvements of the deposition and optical monitoring techniques [1,2], it is now possible to fabricate more complex multilayer structures having up to several hundreds of layers. Most of the focus on the recent developments has been placed on the precise control of the intensity transmitted or reflected by these elements, but very little efforts has been placed in the control of the reflected phase except for the control of group delay dispersion, especially for short pulse laser applications [3] or for a few specific applications including bandpass filters or mirrors [4-7]. In addition, there is higher demand for larger optical elements, which required large efforts in order to improve the uniformity of optical coatings [8]. However, the decrease of the size of the coated area is also challenging and presents wide interest for example for the development of striped filters (e.g. for bolometers [9]), pixelated filters [10] or for laser beam shaping [11]. In this paper, we present the design, fabrication and characterization of mirrors deposited on half of a fused silica, with high reflectivity ( $>99 \%$ ) and a phase shift between the mirror and the uncoated substrate equal to $\pi / 2$ at $1064 \mathrm{~nm}$ [12]. We also show how this approach can be transferred to micro-mirrors with diameters between 50 and $150 \mu \mathrm{m}$ and study the influence of decreasing the size of the micro-mirrors on the quality of the fabricated elements.

\section{Design, fabrication and characterization of mirrors with controlled phase and amplitude}

The simplest and fastest way to design and fabricate a mirror is the use of a metallic layer on top of a glass substrate. The advantage of such a solution is the fact that a single layer is sufficient. But when it comes to high energy laser applications, such an approach is not optimal and the use of dielectric materials appears as a better solution [13]. Such dielectric mirrors are then obtained using a stack of quaterwave layers with alternated high and low refractive indices. A quarterwave-based mirror made out of $\mathrm{Nb}_{2} \mathrm{O}_{5}$ as a high refractive index material $(\mathrm{H}$,
$\left.\mathrm{n}_{\mathrm{H}}=2.20\right)$ and $\mathrm{SiO}_{2}$ as a low refractive index material $\left(\mathrm{L}, \mathrm{n}_{\mathrm{L}}=1.47\right)$ was designed. Based on the refractive index of these two materials, achieving a reflectivity higher than $99 \%$ at $1064 \mathrm{~nm}$ requires depositing at least 15 layers. In addition, we wanted to control the reflected phase, i.e. the difference between the reflected phase on the mirror and the phase reflected by the glass substrate on top of which the mirror is coated, and make it equal to $\pi / 2$. The need of such $\pi / 2$ phase shift is commonly required for several applications including phase contrast microscopy that was developed by Zernike [14]. By introducing a $\pi / 2$ phase between the incident and the scattered components of the image field, it is possible to convert a phase information into an amplitude image. Such plates can also be used for passively coupling of lasers [15]. To achieve such a phase difference, the design of the mirror had to be optimized.

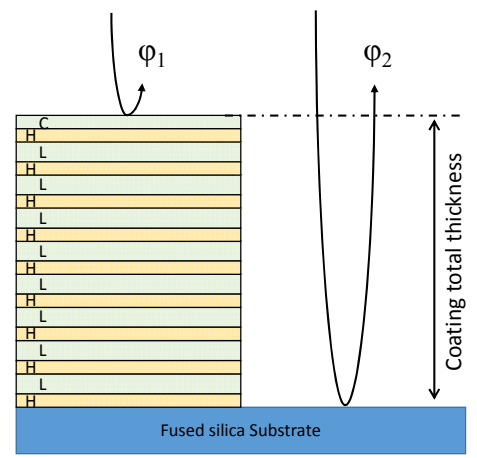

Fig. 1. Definition of the phase reflected by a dielectric mirror and its substrate. Phase is calculated at the level of the dashed line. $\mathrm{H}$ are high refractive index layers, $\mathrm{L}$ are low refractive index layers and $\mathrm{C}$ the phase control layer.

As a matter of fact, a regular quaterwave dielectric mirror exhibits a 0 or $\pi$ phase shift at reflection at its central wavelength depending whether the last layer is a low refractive index material layer (L) or high refractive index material layer $(\mathrm{H})$. In addition, it is possible to obtain any intermediate reflected phase value, between 0 and $2 \pi$, by adjusting the thickness of the last layer of the dielectric mirror ( $\mathrm{L}$ or $\mathrm{H}$ ). In our specific case, a second phase term also appears: the phase 
incursion in air over the mirror-equivalent thickness. To mathematically express this problem and model these phase terms, let us define $\varphi_{1}$, the phase reflected by the mirror at $1064 \mathrm{~nm}$ and $\varphi_{2}$, the phase reflected by the uncoated substrate at the level of the last layer of the coating (Fig. 1). We can write that:

$$
\varphi_{1}=\arctan \left(\frac{\operatorname{Im}\left[\eta_{0}\left(B C^{*}-C B^{*}\right)\right]}{\left(\eta_{0}^{2} B B^{*}+C C^{*}\right)}\right)
$$

where $\mathrm{B}$ and $\mathrm{C}$ denote the appropriate normalized electric and magnetic fields at the air/coating interface and $\eta_{0}$ is the incident substrate admittance [16]. We have also:

$$
\varphi_{2}=\pi\left(1-\frac{4}{\lambda}\left[\left(\sum t_{H}+\sum t_{L}\right)+t_{C}\right]\right)
$$

where $\Sigma t_{H}$ is the sum of the physical thicknesses of the high refractive index layers, $\Sigma t_{L}$ is the sum of the physical thicknesses of the low refractive index layers, and $t_{C}$ is the physical thickness of the last phase controlling layers. By adjusting the thickness of this layer, both $\varphi_{1}$ and $\varphi_{2}$ are modified. We plotted in Fig. 2, the evolution of the $\varphi_{1}, \varphi_{2}$ and $\varphi_{1}$ $-\varphi_{2}$ on the total thickness of the coating.
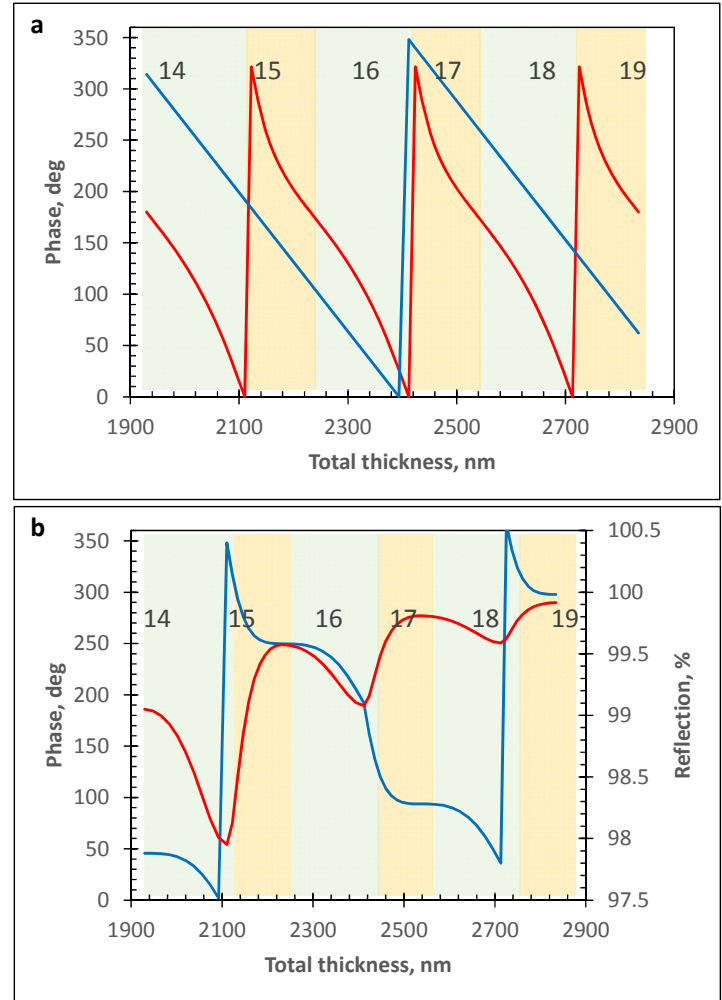

Fig. 2. Figure 2-a. Evolution of the phase reflected by the dielectric mirror (red) and the substrate (blue) on total thickness of the coated mirror (for the last 6 layers of the mirror).

Figure 2-b. Evolution of the difference of phase $\varphi 1$ - $\varphi 2$ (red) and the reflected amplitude (blue) on total thickness of the coated mirror.

For both Figures, green rectangles show the low refractive index layers position and the orange rectangles show the position of the high refractive index materials, starting from a 13-layer mirror and ending with a 19-layer mirror.

One can see that achieving a reflection higher than $99 \%$ on the mirror combined with a $\pi / 2$ phase shift difference $\left(\varphi_{1}-\varphi_{2}\right)$ is achieved only once in the graph of Figure 2, i.e. after deposition of a 17-layer quaterwave mirror followed by a $50 \mathrm{~nm}$ thick silica layer. In addition, one can see that the phase difference is close to $90^{\circ}$ after depositing the 17-layer mirror and that the $50 \mathrm{~nm}$ silica layer allows fine tuning the phase. This adjustment could probably be neglected, but we decided to take it into account in our fabrication process in order to secure a perfect theoretical phase. Also, in this range, as the phase is slowly varying, the precise control of this last layer is not critical and small thickness errors would only result in a few degrees error on the phase difference.

To validate the approach, we fabricated a prototype of such an element by physically masking half of a fused silica substrate and depositing a mirror on the other half. Deposition was achieved using a Bühler SYRUSpro 710 machine associated with an OMS 500 optical monitoring system. As it is critical to be able to precisely control both the total physical and the optical thickness of the mirror, we opted for an optical monitoring of the mirror during deposition. While the parameter controlling the phase reflected by the mirror at $1064 \mathrm{~nm}$ is the total optical thickness of the mirror and can be well controlled using a classical turning point optical monitoring strategy due to the well-known compensation of errors phenomenon, such an effect would however affect the total physical thickness of the stack and therefore the phase reflected on the uncoated side. To overcome this problem, we opted for a trigger point monitoring technique on one edge of the mirror (i.e. $\sim 940 \mathrm{~nm}$ ). This technique allows minimizing the errors on the thickness of each individual layer. In addition, it allows maintaining a large amplitude of the monitoring signal, even when the mirror becomes highly reflective. After fabrication, the spectral dependence of the reflection was characterized using a Perkin Elmer Lambda 1050 spectrophotometer equipped with an URA module that allows absolute measurement of the reflectivity. Accuracy of such high reflection coefficient measurement was tested using a calibration sample having more than $99.95 \%$ reflection at $1064 \mathrm{~nm}$. A reflectivity exceeding $99 \%$ and equal to $99.7 \pm 0.2 \%$ was confirmed (Figure 3). One can also see a good theory/experiment agreement.

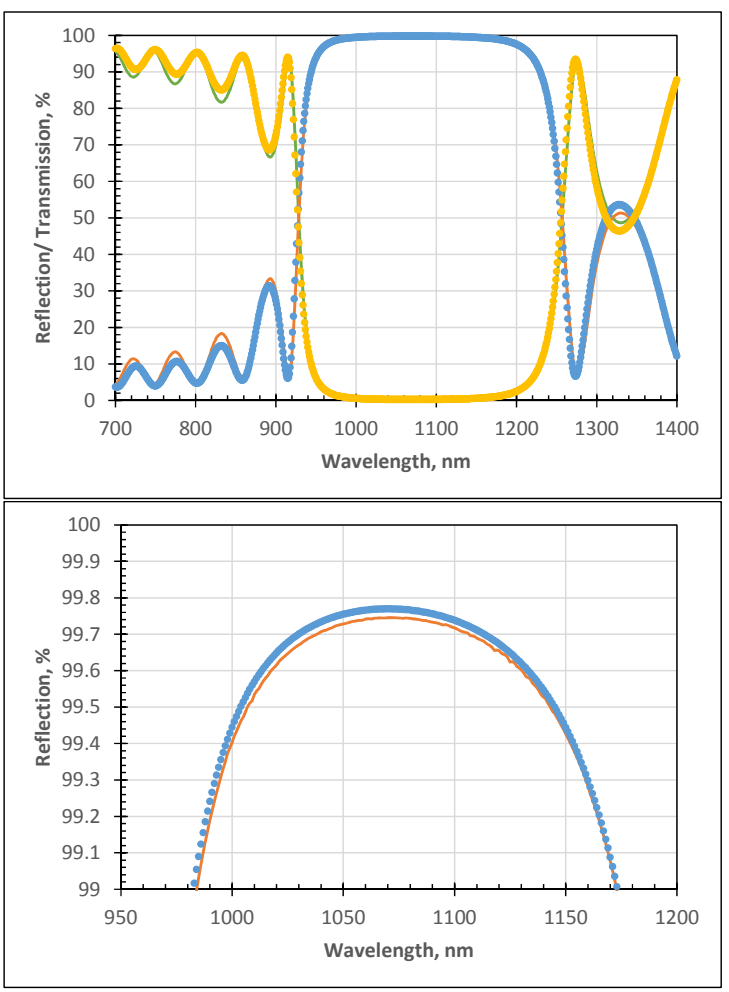


Fig. 3. Spectral dependence of the transmission (thin green: theory, thick yellow: experiment) and the reflection (thin orange: theory, thick blue: experiment) of the fabricated mirror.

To characterize the phase difference between the mirror and the uncoated substrate, we developed a dedicated setup. For this phase measurement, the geometry chosen is a geometry of a Michelson interferometer (Fig. 4). The laser source is a laser diode fiber $1064 \mathrm{~nm}$ from Innolume with a spectral width of $0.1 \mathrm{~nm}$ and single-longitudinal mode. The beam issuing from the fiber is collimated with a diameter of $7 \mathrm{~mm}$. A pellicle beam splitter is used to separate the beam into two new beams. On one arm of the interferometer a reference mirror is placed to reflect back the beam while the other arm is used for the measurement of the bizone plate. Finally, the beams are recombined onto a CCD camera. It should be noted that a lens is used to image the bi-zone plate and the mirror on the CCD camera and thus reduce the diffraction problems that occur at the interface between the mirror and the uncoated area. Laser power control, camera-level acquisition, and signal processing are achieved through programs developed under LabVIEW.

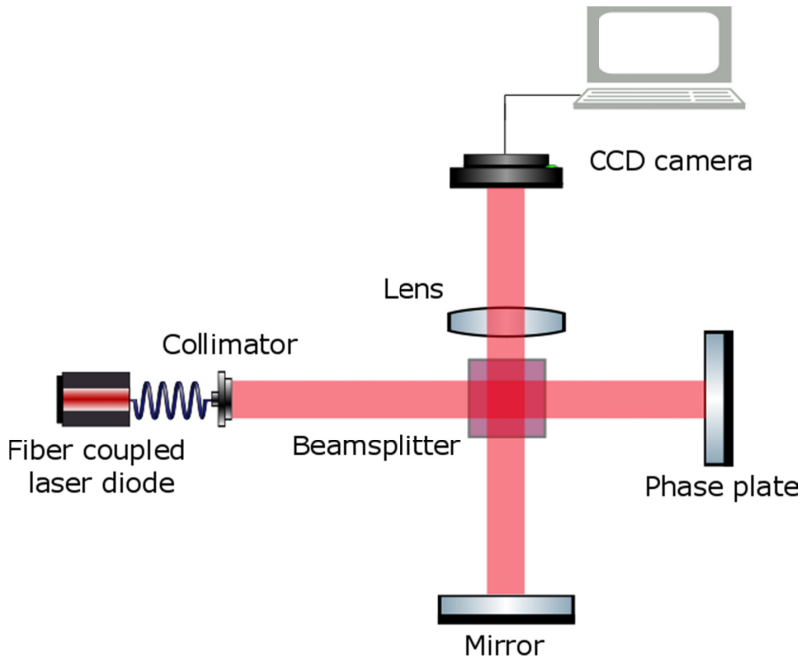

Fig. 4. Optical system developed for the measurement of the phase difference between coated and uncoated substrate.

During a measurement, the sample is placed so that the mirror/glass transition is horizontal and the virtual angle between the two mirrors is adjusted so that the produced fringes are orthogonal to this transition. Thus, on each of the lines of the camera, the intensity equals:

$$
I(x)=I_{1}+I_{2}+2 \sqrt{I_{1} I_{2}} \cos \left(\frac{2 \pi}{\lambda} \Delta t(x)+\varphi\right)
$$

Where $I_{1}$ and $I_{2}$ are the intensities reflected by the 2 components, $\lambda$ the wavelength, $\Delta t(x)$, the optical path difference (linearly variable according to $\mathrm{x}$ ) between the 2 arms of the interferometer and $\varphi$ the phase shift at reflection which equal $\varphi_{1}$ on the mirror and $\varphi_{2}$ on the uncoated substrate. By comparing the phase before and after the transition, it is thus possible to measure the phase shift $\varphi_{1}-\varphi_{2}$

Figure 5 describes the method that has been developed for the calculation of the phase shift $\varphi_{1}-\varphi_{2}$ from the interferogram. The intensity graph shows the uncoated area at the top and the coated area at the bottom. The visibility of the interference fringes is different because of differences in reflection coefficients, but this difference does not affect the phase calculation. For a given horizontal line, it is possible to extract the intensity fluctuations versus $\mathrm{X}$ (pixel number) that follows equation (3). From this signal, it is then possible to calculate its Fourier transform or do a cross-correlation between two successive lines and extract two information, i.e. the frequency of the signal, that is the same (or close) for all lines and depends on the virtual tilt between the two mirrors (the higher the tilt, the higher the frequency), and the second information is the phase $\varphi$ of equation (3).

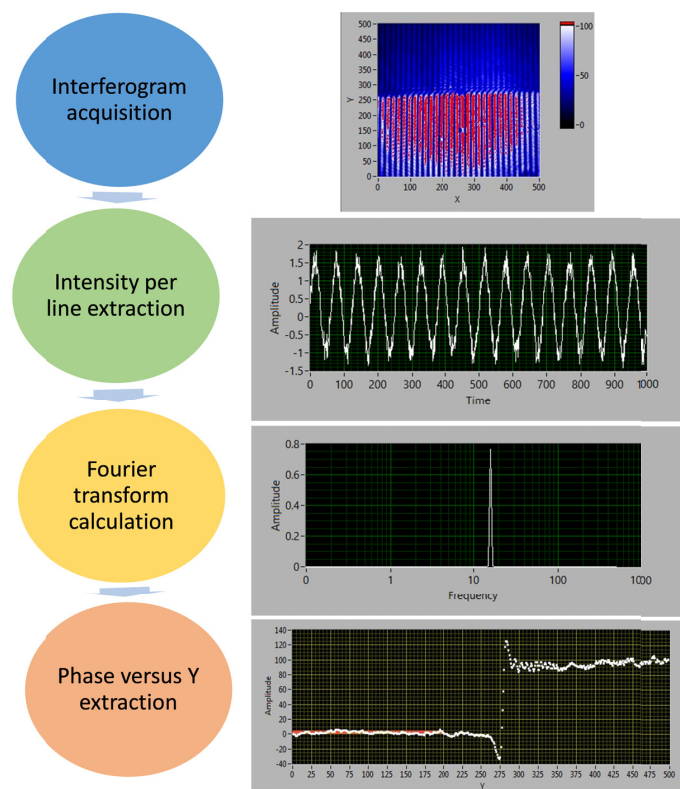

Fig. 5. Principle of the phase calculation method.

By applying this technique for each of the line, it is then possible to extract the evolution of the phase $\varphi$ on Y (pixel number). Such an approach is only possible because we suppose that for a given line $\mathrm{X}$, the phase is the same along the whole line (this is true for plane substrate) and because the transition between coated and uncoated zone is occurring over a narrow spatial region. Then, for the uncoated zone, the phase is supposed to be the same all over the sample surface. By properly aligning the fringes to have them vertical, it is possible to cancel any wavefront tilt. The value of $\varphi_{1}$ is then calculated by averaging the phase in a coated region close to the transition and value of $\varphi_{2}$ is then calculated by averaging the phase in an uncoated region close to the transition.

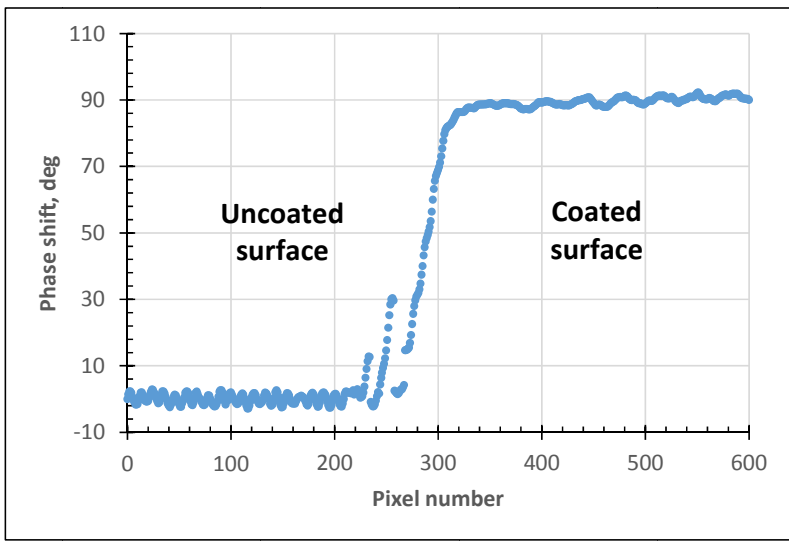

Fig. 6. Phase difference measured on the fabricated sample.

Figure 6 shows the evolution of the phase along the $Y$ axis (pixel number) obtained by cross-correlation between the signal I(x) (according to equation [3]) for $\mathrm{Y}=0$ and the signal $\mathrm{I}^{\prime}(\mathrm{x})$ for each $\mathrm{Y}$ value between 0 and $N$ (the total number of lines along $Y$ ). For $Y=[0,200]$, the measured phase is the one of coated surface $\left(\varphi_{1}\right)$. We supposed that at $Y=0$, this phase is equal to zero (reference phase) and then 
fluctuates around $0^{\circ} \pm 3^{\circ}$ due to noise measurement. For $Y=[350,600]$ the measured phase is the one of the uncoated surface $\left(\varphi_{2}\right)$ with respect to the phase at $Y=0$ and is equal to $90^{\circ} \pm 3^{\circ}$. For $Y=[250,350]$ (i.e. over $\sim 500 \mu \mathrm{m}$ ), the measured phase, with respect to the one at $Y=$ 0 , varies from $0^{\circ}$ to $90^{\circ}$ with some sharp fluctuations related to the diffraction at the interface but also to a non-perfectly straight transition. Hence, using this approach, we could confirm that the phase difference between the mirror and the uncoated face is equal to $\pi / 2 \pm \pi / 20$.

\section{Fabrication of micro-mirrors with controlled amplitude and phase}

We were not only interested in fabricating a bi-zone plate with different reflected amplitudes and phases, but also by controlling the size and shape of the mirrors in order to produce circular mirrors with diameter of a few tens of microns. For this purpose, we used classical photolithography based on lift-off technique following these 5 different steps:

1. Spin coating was first used to deposit a photoresist on top of a glass substrate (a).

2. Structured illumination was then performed through an amplitude mask (b). This amplitude mask allowed reproducing a sequence of circular shapes with different diameters ranging from 50 to $125 \mu \mathrm{m}$.

3. Photoresist was developed in order to remove it where exposure was performed (c). All the wafer was therefore covered by a photoresist film except in the cylindrical openings produced by the mask.

4. The phase mirror was then deposited on top of this substrate (d).

5. Lift-off of the photoresist was performed in order to remove the coating all over the substrate, in the parts where photoresist was present (e).

One can note that if single photoresist is applied, there would be material deposition on the side of the photoresist mask resulting in walls creation and a wrong shape of each of the mirrors. Such an effect would alter the uniformity of the mirrors over their aperture especially close to the edges of the mirrors, increasing the transition zone. To overcome this problem, a bilayer masking solution was implemented (Figure 7) [17]

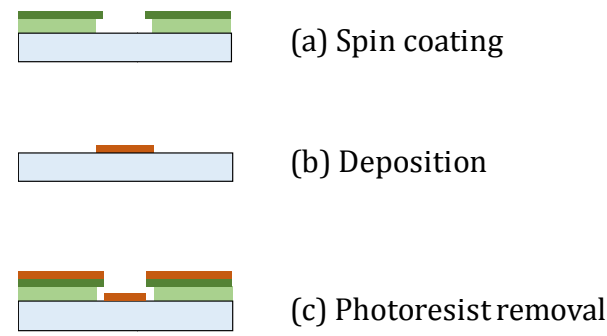

Fig. 7. Photolithographic approach with two photoresists.

It uses the combination of two photoresists with different etching properties. The first one is dedicated to creating the main mask pattern and the second one is then added in order to create cap-like profiles that will prevent side-wall deposition. Using this technique, it is therefore possible to fabricate small mirrors with sharp edges and uniform properties. All photolithographic processes were performed by the SILIOS Company.

The deposition of the filter was obtained by plasma-assisted electron beam deposition using the Bühler SYRUSpro 710 machine. The deposition were carried out within the thin film facility of the Institut Fresnel. Similarly to the bi-zone plate, high refractive index material was $\mathrm{Nb}_{2} \mathrm{O}_{5}$ while the low refractive index materials was $\mathrm{SiO}_{2}$. The design of the mirror was identical to the one of section 2, i.e. with total thickness of each of the mirrors within $2.6 \mu$ m, i.e. 20 times thinner than the smaller aperture. These mirrors were optically monitored during deposition with the same technique as presented in section 2 . Optical monitoring was not performed directly on the micro-mirrors but on a witness sample placed in the sample holder next to the micromirrors. A bi-zone plate was also placed in the chamber for direct phase measurements. Preliminary tests showed that the spectral response obtained on the witness samples is within the measurement errors, identical to the one of the structured filters [18].

To confirm that these micro-mirrors have the requested performances, we performed, after fabricating, various characterizations. First, we measured the geometric properties of the fabricated mirrors with the use of a Zygo NewView 7300 and 10x objective (Figure 8). Similarly to what was presented in Ref. [10], annealing of the photoresist due to process temperature and interaction with the assistance plasma could be observed, but this effect was limited by using low temperature deposition. This was achieved by limiting the temperature during the deposition to $100^{\circ} \mathrm{C}$ and pausing the deposition each time temperature is approaching this limit temperature.

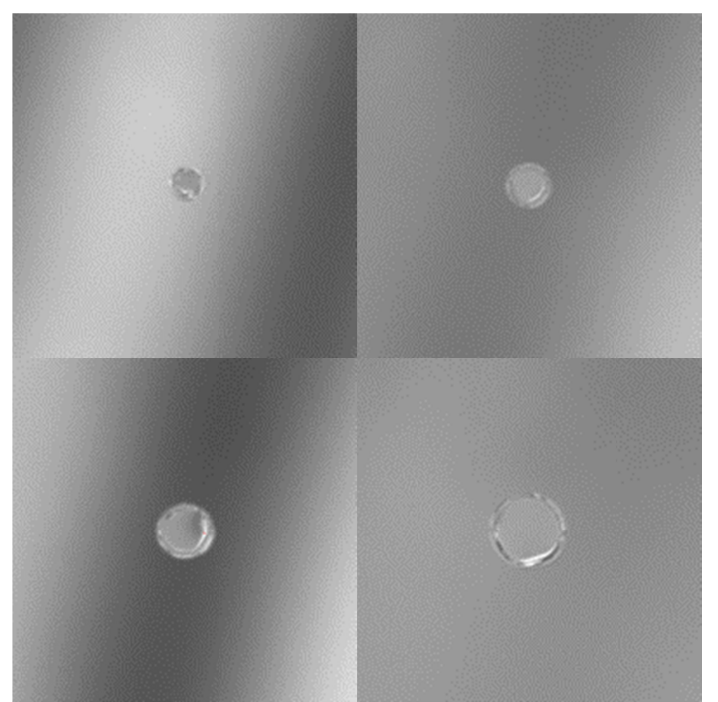

Figure 8. Interferograms of the micro-mirrors as measured by white light profilometry. From Left to right, increasing mirror diameter: 50, 70,90 and $125 \mu \mathrm{m}$.

One can also see that all the mirrors present a transition zone equal to $5-10 \mu \mathrm{m}$ at the periphery of the micro-mirrors, which is due to the masking associated with the deposition. In the center part, one can see that the mirrors appear pretty uniform, confirming that the masking technique did not affect too much the deposition uniformity. Finally, one can see that there are some defects of the mirror shape. These effects can be attributed to the photoresist annealing occurring the mirror deposition. Exact analysis of the geometrical shape of these mirror is a challenging task as large diffraction occurs at the boundary of the mirror when measurement is performed. Therefore, for the smaller mirrors, only qualitative analysis of the mirrors as the one provided by Figure 8 could be performed as the Zygo Newview could not precisely calculate the mirror profiles. However, quantitative analysis could be done for the larger mirrors (Figure 9).

One can see that the fabricated mirrors are very uniform over $80 \%$ of the aperture. As stated and visible on the interferograms, the transition 
zone appears pretty sharp, not exceeding 5-10 $\mu \mathrm{m}$. Small defects at the boundary however create small local non-uniformities.

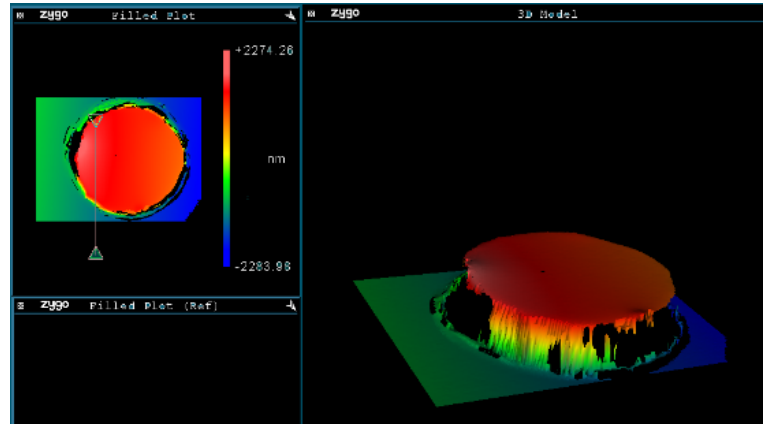

Figure 9. Spatial profiles of the $125 \mu \mathrm{m}$ micro-mirrors as measured by white light profilometry.

Phase and amplitude of the reflection at $1064 \mathrm{~nm}$ of the micro-mirrors could not directly be measured. However, we first indirectly characterized these properties on the witness sample similar to the one presented in section 2 and that was coated simultaneously with the micro-mirrors. Table 1 presents the performances measured on the witness samples of section 2 and 3 .

Table 1. Performances measured on the witness sample

\begin{tabular}{|c|c|c|c|}
\hline Sample name & Lambda $\mathbf{R}_{\max }$ & $\mathbf{R}_{\max }$ & $\boldsymbol{\varphi}_{\mathbf{1}}-\boldsymbol{\varphi}_{\mathbf{2}}$ \\
\hline Sample section 2 & $1075 \mathrm{~nm}$ & $99.7 \pm 0.2 \%$ & $90^{\circ} \pm 3^{\circ}$ \\
\hline $\begin{array}{c}\text { Witness sample } \\
\text { section 3 }\end{array}$ & $1076 \mathrm{~nm}$ & $99.7 \pm 0.2 \%$ & $90^{\circ} \pm 3^{\circ}$ \\
\hline
\end{tabular}

One can see that due to the high repeatability of the fabrication processes of the SYRUSpro 710 machine associated with the direct optical monitoring, performances of the witness sample are comparable to the one presented in section 2 . These result show that the deposited structure was the expected one.

In order to validate that the micro-mirrors have identical properties, transmission spectra was directly measured on the micro-mirrors measured using an internal system based on an optical spectral analyzer that is generally used to characterize the uniformity of optical filters [19]. Using a $50 \mu \mathrm{m}$ beam diameter, transmission was measured on the $125 \mu \mathrm{m}$ micro-mirror and compared to the one on the witness sample (Figure 10).

One can see that the two measured transmission spectra are very close. Higher noise is measured on the micro-mirror due to the lower energy level in this measurement. These data prove that masking did not affect the coating at least in the central part and confirm the one of previous studies that showed that the use of a photoresist mask does not affect the spectral properties of the deposited components compared to un-masked one $[10,18]$. One can wonder if this verification is enough to secure that both amplitude and phase properties are met. However, it is important to note that only dielectric materials with no measurable absorption at $1064 \mathrm{~nm}$ were used for the fabrication of the mirrors, securing minimal losses in the mirror and a reflection coefficient equal to $1-\mathrm{T}$ where $\mathrm{T}$ is the measured transmission. The low average transmission around $1064 \mathrm{~nm}$ below $1 \%$ confirms a reflection coefficient larger than $99 \%$. In addition, it is interesting to note that the very good matching of the transmission spectral measurements over a broad spectrum (Figure 10), also secures an optimal value of the phase $\varphi_{1}-\varphi_{2}$ as any fabrication errors that would affect the phase value would also affect the transmitted intensity.

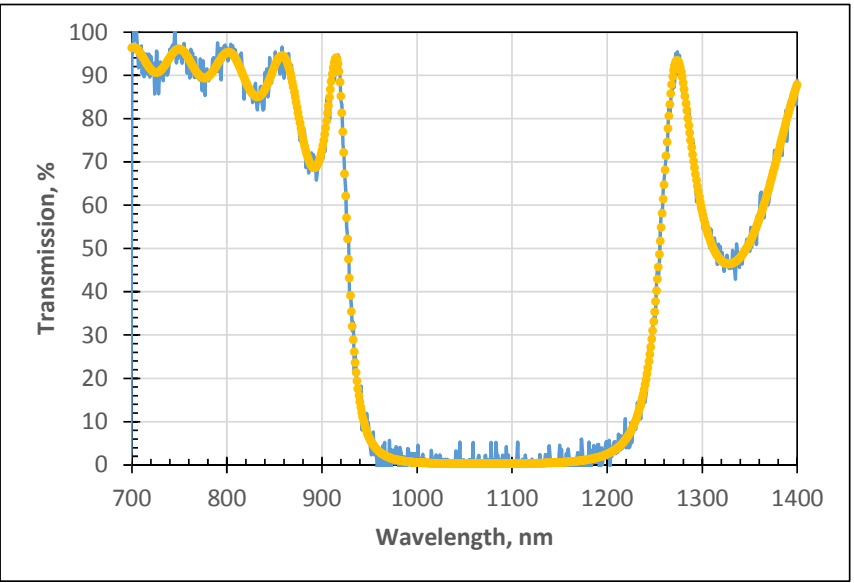

Figure 10. Spectral dependence of the transmission. Blue: measurement on the $125 \mu \mathrm{m}$ micro-mirror, yellow: measurement on the witness sample.

As an example, adding a $50 \mathrm{~nm}$ thick silica layer on top of the 17-layer mirror as the one used to finely tune the reflected phase shifts the minimum of transmission from 1064 to $1075 \mathrm{~nm}$. These various characterizations therefore demonstrate that we were able to fabricate micro-mirrors with the expected properties. Finally, it is worth noting that the validity of the performances of the components could finally be indirectly verified by integrating the component in a laser system and verifying that it acts as expected.

\section{Conclusions}

Micro-mirrors with controlled phase and amplitude have been demonstrated. These mirrors were obtained by combining proper design of both the amplitude and the phase reflected by these elements and shaping using photolithographic process. These results pave a way towards more complex elements, especially for laser beam shaping by diffractive optical elements.

Acknowledgment. The Perkin Elmer Lambda 1050, the ZYGO NewView 7300 and BUHLER Optics SYRUSpro 710 machine were acquired within the framework of the Espace Photonique facility and funded by the financial support of the French Department of Industry, AMU, CNRS, the local administrations (Provence-Alpes Côte d'Azur Regional council, Bouche du Rhône council, town of Marseille), and the European Community.

\section{References}

1. M. Scherer, J. Pistner and W. Lehnert, "UV- and VIS Filter Coatings by Plasma Assisted Reactive Magnetron Sputtering (PARMS)," OIC 2010 Proceedings.

2. A. Zoeller, M., H. Hagedorn, W. Klug and C. Schmitt, "High accurate insitu optical thickness monitoring," OIC 2004 Proceedings.

3. E. Fedulova, K. Fritsch, J. Brons, O. Pronin, T. Amotchkina, M. Trubetskov, F. Krausz, and V. Pervak, "Highly-dispersive mirrors reach new levels of dispersion," Opt. Express 23, 13788-13793 (2015).

4. A. Piegari, G. Emiliani, "Laser mirrors with variable reflected intensity and uniform phase shift: design process", Appl. Opt. 32, 5454-5461 (1993).

5. F. Lemarquis, E. Pelletier, "Optical coating without phase dispersion for a Fabry-Perot interferometer", Appl. Opt. 35, 4987-4992 (1996). 
6. A.V. Tikhonravov, P. W. Baumeister, K.V. Popov, "Phase properties of multilayers", Appl. Opt. 36, 4382-4391 (1997).

7. W. Shen, X. Liu, B. Huang, Y. Zhu, P. Gu, "The effect of reflection phase shift on the optical properties of a micro-opto-electro-mechanical system Fabry-Perot tunable filter" J. Opt. A: Pure Appl. Opt. 6 , 853858, (2004).

8. J.-M. Mackowski, L. Pinard, L. Dognin, P. Ganau, B. Lagrange, C. Michel, C., and M. Morgue, "VIRGO Mirrors: Wavefront Control," Springer Verlag (Germany), 1, 1999.

9. R. Le Goff, B. Badoil, P. Fuss, F. Tanguy, P. Etcheto, "Recent developments of multispectral filter assemblies for CCD, CMOS and bolometer," Proc. SPIE 8176, Sensors, Systems, and Next-Generation Satellites (2011).

10. M. Lequime, L. Abel-Tibérini, K. Mathieu, J. Berthon and J. Lumeau, "2×2-array pixelated optical interference filters", Proc. SPIE 9627-30 (2015).

11. Y. Xie, Z. Lu, F. Li, J. Zhao, and Z. Weng, "Lithographic fabrication of large diffractive optical elements on a concave lens surface," Opt. Express 10, 1043-1047 (2002).

12. J. H. Lumeau, C. Koc, and T. Begou, "Micro-mirrors with controlled amplitude and phase," in Optical Interference Coatings 2016, OSA Technical Digest (online) (Optical Society of America, 2016), paper FA.10.

13. K V S R Apparao, "Dielectric mirrors for high power laser applications," Bulletin of Materials Science 8(3), 339-342 (1986).

14. Zernike F. How I discovered phase contrast. Science 121, 345-349 (1955)

15. H.-S. Chiang J. R. Leger, "Uncovering the physical origin of self-phasing in coupled fiber lasers", Proc. SPIE 9343, Laser Resonators, Microresonators, and Beam Control XVII, 93431A (2015)

16. H. Angus Macleod, Thin-Film Optical Filters, Fourth Edition, CRC Press

17. Harry J. Levinson, Principles of Lithography, SPIE Press, Third Edition (2010).

18. S. Sorce, L. Abel-Tiberini, and M. Lequime, "Spectrophotometric bench dedicated to the characterization of micro-patterned optical coatings," Proc. SPIE Col. 8169, 81690N-1 (2011)

19. L. Abel-Tiberini, F. Lemarquis and M. Lequime, "Dedicated spectrophotometer for localized transmittance and reflectance measurements," Appl. Opt., 45, 1386-1391 (2006) 